\title{
Parkinson's Disease Gravity Index: A Method by means of Optimal Scaling
}

\author{
Marcos Serrano-Dueñas (D), ${ }^{1,2}$ Luis Masabanda, ${ }^{2}$ and Maria-Rosario Luquin (i) $^{3}$ \\ ${ }^{1}$ Facultad de Medicina, Pontificia Universidad Católica del Ecuador, Quito, Ecuador \\ ${ }^{2}$ Servicio de Neurología, Hospital Carlos Andrade Marín, Quito, Ecuador \\ ${ }^{3}$ Departamento de Neurología, Clínica Universidad de Navarra, Pamplona, Spain \\ Correspondence should be addressed to Marcos Serrano-Dueñas; mserranod@puce.edu.ec
}

Received 17 August 2020; Revised 11 November 2020; Accepted 4 December 2020; Published 16 December 2020

Academic Editor: Vincenzo Di Lazzaro

Copyright (C) 2020 Marcos Serrano-Dueñas et al. This is an open access article distributed under the Creative Commons Attribution License, which permits unrestricted use, distribution, and reproduction in any medium, provided the original work is properly cited.

\begin{abstract}
Objective. This study has been designed with the aim of using optimal scaling to perform the allocation of scores and to be able to construct an indicator of the Parkinson's Disease Gravity Index. Scores were assigned to interrelated dimensions that share information about the patient's situation, to have an objective, holistic tool which integrates scores so that doctors can have a comprehensive idea of the patient's situation. Patients and Methods. 120 consecutive patients with Parkinson's diagnosis were chosen according to the United Kingdom Parkinson's Disease Society Brain Bank criteria. Subsequently, all the chosen dimensions were transformed into interval variables for which the formula proposed by Sturges was used. Once the dimensions were transformed into interval variables, optimal scaling was carried out. Subsequently, the following attributes were analyzed: quality and acceptability of the data; reliability: internal consistency, reliability index, Cronbach's alpha, and standard error of measurement; finally, validity: convergent validity and validity for known groups. Results. There were no missing data. An appropriate Cronbach's alpha value of 0.71 was gathered, and all items were found to be pertinent to the scale. The item homogeneity index was 0.36 . Precision evaluated with the standard error of measurement was 7.8. The Parkinson's Disease Gravity Index discriminant validity (validity for known groups), assessed among the different stages of Hoehn and Yahr scale by the Kruskal-Wallis test, showed major significance $\left(X^{2}=32.7, p \leq 0.001\right)$. Conclusions. The Parkinson's Disease Gravity Index has shown adequate metric properties.
\end{abstract}

\section{Introduction}

Parkinson's disease (PD) is a heterogeneous neurodegenerative process, which, by 2016, was estimated to affect some 6.1 million people [1].

The onset of neurodegeneration of Parkinson's disease is likely to occur several decades before the onset of motor symptoms. Possible risk factors include genetic predisposition and environmental factors such as exposure to toxins. Parkinson's disease is characterized by a selective loss of dopaminergic neurons in the substantia nigra pars compacta; subsequently, there is a widespread involvement of other circuits of the central and peripheral nervous system. Changes associated with genomic, epigenetic, and environmental factors lead to structural alterations and protein deposits, especially alpha-synuclein, due to dysfunction in the ubiquitin-proteasome system, alteration of mitochondrial function, and oxidative stress [2].

$\mathrm{PD}$ is more than just a movement disorder. Other PD subtypes have been proposed, such as autonomic dysfunction, cognitive deterioration, and REM sleep disorder. However, there is still a clear emphasis on the motor symptoms associated with PD [3]. There are different degrees of nonmotor symptoms of the disease (NMS) such as autonomic dysfunction, sensory disorders, integumentary system disorders, neurobehavioral disorders, sleep disorders, visual impairment, and other conditions [4].

We should point out that there is no clear correlation between motor involvement and nonmotor disorders. In a study of 935 patients, Ray Chaudhuri et al. [5] concluded 
that although the NMS (Nonmotor Symptoms Scale (NMSS)) [6] increases with the severity of PD, the correlation between motor disorder and the NMS was moderate (Spearman's $\rho($ rhoS $=0.43)$ ). This shows that the association between motor disorder and NMS varies at all stages of the disease, except for in the most advanced cases. Most importantly, there is a clear incongruity between motor and nonmotor abnormalities as patients in the milder stages of motor disorder may have considerable nonmotor symptoms. For example, in the study by Ray Chaudhuri et al. [5], more than a third of the patients (34.5\%) were in stages 1 and 2 of the Hoehn and Yahr (H\&Y) scale [7] and presented severe or very severe nonmotor symptoms.

The International Parkinson and Movement Disorders Society offers MDS-owned rating scales, translated scales, and a listing of other recommended rating scales to carry out the evaluation of all the previously mentioned conditions. Using these scales, by the end of the evaluation of patients, a series of scores of different amounts are generated.

These scores should be considered by the doctor. However, the doctor, in a personal and subjective manner, should assign their own scores based on their experience, with the goal of reaching a total score which is a comprehensive view of the situation of the patient, and create an appropriate treatment plan. There is a published proposal to perform the Parkinson's Disease Composite Scale (PDCS) that uses heterogeneous values to assess and categorize the severity of symptoms [8]. The authors used the disease staging according to the Hoehn and Yahr (H\&Y) scale and various scores in sections of the Clinical Impression of Severity Index for Parkinson's Disease (CISI-PD): the Movement Disorder Society-Unified Parkinson's Disease Rating Scale (MDS-UPDRS), the Nonmotor Symptoms Scale (NMSS), or the Nonmotor Symptoms Questionnaire (NMSQuest).

However, a core issue remains unanswered: how should doctors assess the importance of symptoms? There are such diverse symptoms which belong to quite different fields, for example, the anxiety section of the HADS (a construct, evaluated by the patient himself) which has a maximum score of 21 points and the motor SCOPA (a scale based on the examiner's scores) which has a maximum score of 75 . If the patient scores 7 points of anxiety, is it more (or less) important than 25 points of motor SCOPA? Similar questions can be asked of the other scales.

On the other hand, we must assume that the scores of the scales are actually discrete scores, that is, that in a strict sense, they do not admit fragmentation and only positive integers belonging to the set of real numbers (natural numbers) are possible, and therefore, they could not be divided.

Given the dispersion of the scores, we should treat them as interval variables (class intervals) (by classifying, ordering, establishing a distance between the values, and having a unit of measurement). In this type of measurement, the numbers assigned to the objects have all the characteristics of ordinal measurements, and the differences between the measurements represent equivalent intervals. Once these intervals have been created, we can consider them as ordinal categorical variables (classification and sorting), which must also be exhaustive, exclusive, and referenced to a single classifying principle [9].

Due to this, it is necessary to have a mathematical algorithm that allows us to take all the scores and transform them so that, in the end, we have comparable scores.

Fisher called this methodology "the appropriate scoring technique" [10]. Later, Young made the optimal scaling (OS) proposal $[11,12]$ as the appropriate mathematical method for transforming scores.

Briefly, if we have a matrix $\mathbf{X}$ that contains $\mathbf{n}$ individuals and $\mathbf{m}$ variables, that is to say, a matrix $\mathbf{n} \times \mathbf{m}$, OS is a multivariate analysis technique, which analyses the relationship of the $\mathbf{n}$ random variables and allows for reducing the size of the data matrix with a minimum loss of information. Many of those $\mathbf{m}$ variables are categorical in nature (nominal or ordinal). The idea is to assign quantitative values to these categorical variables using nonlinear methods since not all $\mathbf{m}$ variables are linearly correlated with each other.

Since, in practice, multivariate systems never have maximum homogeneity (variables can share information, but not totally), the reduction of dimensions always involves loss of information, which can be measured through a loss function. The functions most used for this purpose are made by averaging the sums of the squares of the differences between the score vector of the individuals and each of the variables.

This is equivalent to gaining the vector $\mathbf{Y}$ as the summary of the variables previously scaled or linearly transformed, so that each is weighted according to the amount of information shared with the other variables. The transformations that guarantee that the summary vector (score vector of the individuals) collects as much information as possible are usually gained based on the breakdown of values and eigenvectors of the matrix [13-15].

For this reason, this study has been designed with the aim of using OS to perform the allocation of scores and to be able to construct an indicator of the Parkinson's Disease Gravity Index (PDGI). The study's objective is to assign scores to interrelated dimensions that share information about the patient's situation, in order to have an objective, holistic tool which integrates scores so that doctors can have a comprehensive idea of the patient's situation.

\section{Patients and Methods}

To calculate the sample size, parameters suggested by Beavers et al. [16] were applied, and the UKPDSBB clinical diagnosis criteria [17] were used to select the one hundred and twenty patients with PD who participated in the study. All patients were attended at the Ambulatory Setting of the Unit of Abnormal Movements of the Neurology Service of the Carlos Andrade Marín Hospital, Quito, Ecuador.

All patients gave their informed consent to participate in the study, which was approved by the Teaching and Research Department of the Carlos Andrade Marín Hospital and by the Bioethics Committee of the University of Navarra (Spain). 
The exclusion criteria involved the presence of any neurological disorder that caused disability: hemiplegia, blindness, deafness, or the presence of a serious acute illness.

Patient Evaluations. All patients were evaluated during the "ON" period.

The following dimensions were included in the evaluation: (1) age dimension (AD) in years, (2) motor dimension (MD) evaluated with SPES-SCOPA [18], (3) depression dimension (DD) evaluated with HADS [19], (4) anxiety dimension $(\mathrm{AxD})$ with the same, (5) cognitive dimension (CD) evaluated with PD-CRS [20], (6) apathy dimension (ApD) measured with AS [21], (7) fatigue dimension (FD) with D-FIS [22], (8) nonmotor dimension (NMD) with the NMSS [23] (except for the following domains: sleep, fatigue, mood, apathy, perceptual problems, hallucinations, attention problems, and memory), (9) psychosis dimension (PsD) measured with SCOPA-PC [24], and (10) sleep dimension (SD) evaluated with SCOPA-SLEEP [25].

Demographic data of interest were collected. Besides the rating scales indicated, the stages of the disease were evaluated using the H\&Y scale [26]. The Schwab and England (S\&E) scale was used to study daily life activities [27]. The PIMS was used to assess the quality of life [28], and finally, the CISI-PD [29] was used to make clinical information of the severity of the disease.

2.1. Method of Analysis. The PDGI contains the 10 dimensions indicated above: (1) age dimension (AD), (2) motor dimension (MD), (3) depression dimension (DD), (4) anxiety dimension (AxD), (5) cognitive dimension (CD), (6) apathy dimension $(\mathrm{ApD}),(7)$ fatigue dimension $(\mathrm{FD}),(8)$ nonmotor dimension (NMD), (9) psychosis dimension (PsD), and (10) sleep dimension (SD).

Descriptive statistics of the central tendency and dispersion were gathered. Subsequently, all the chosen dimensions were transformed into interval variables, for which the formula proposed by Sturges [30] was used: $K=R /$ $(1+3,322 * \log N)$, where $K$ is the number of intervals, $R$ is the range, and $\log N$ is the natural logarithm (base 10) of $N$, which is the number of individuals. Then, we calculated $W=K / R$, where $W$ is the width/width of each interval.

Once the dimensions were transformed into interval variables, OS was carried out. For this, the statistical package SPSS.v.17 was used.

After retrieving the initial values of the intervals, OS was carried out, which assigns a value to each interval. We collected the minimum (the value of which is absolute) and added to each of the previous values, and we were left with what we call the "corrected value of each interval."

From each dimension, we took the "maximum corrected value of each interval" and added them. In our case, there were 10 dimensions, the sum of which reached 36.44 . Since we know that the maximum theoretical value is 100 , we divided it by that sum $(100 / 36.44)=2.744$, which is the "index factor."
We multiplied the "index factor" by each of the "corrected values of each interval" and thus found the new "corrected values multiplied by the factor." For confirmation, if we add up the "maximum corrected values multiplied by the factor," we will get 100 (model) (Table 1).

For example, for a patient who has an original score with the SPES-SCOPA in the interval of 24-30 points, the corrected value is 1.18 . When multiplied by the factor of 2,744 , the final value is 3.24 points, which is the number used in the PDSI (Table 2).

2.1.1. Data Quality and Acceptability. (i) Lost data must not exceed 5\%; (ii) the difference between the average and median should not exceed $10 \%$ of the highest possible score; and (iii) the floor and ceiling effects must not exceed 15\% [31]. The skewness and kurtosis coefficients must lie within the interval of $-1,1[32]$.

2.1.2. Reliability. (i) Internal consistency: the homogeneity index of the items must be $\geq 0.330$; and (ii) the reliability index, Cronbach's alpha (C'a) value, must be greater than 0.70 [33], and the standard error of measurement (SEM) was attained; the SEM must be equal to the standard deviation, multiplying by the square root of 1 minus $C^{\prime} \mathrm{a}=(\mathrm{SD} * \sqrt{ } 1-$ reliability coefficient $)$ [34], where SD indicates standard deviation. We compared the SEM with half the amount of the standard deviation and gained a lower value, thus yielding a precision of $\geq 75 \%$ [35].

2.1.3. Validity. (i) Convergent validity: for this, Spearman's correlation coefficient (rhoS) and the values suggested by Akoglu [36] were used $(0=$ no correlation; $0.1-0.3=$ weak correlation; $0.4-0.6=$ moderate correlation; $0.7-0.9=$ strong correlation; $1=$ perfect correlation) and (ii) validity for known groups: for this, we used the H\&Y stages as a segmentation variable, and a value $\leq 0.05$ was observed as significant.

\section{Results}

There were 120 patients with a mean age of 68.5 years, 9 years of illness, with $683.5 \pm 225.5 \mathrm{mg}$ of levodopa/day (or equivalent dose of levodopa); $60.8 \%$ of patients were males. The same number of patients were retired. Seventy-four (61.7\%) were in stage III of the H\&Y classification (Table 3). The mean $\pm(\mathrm{sd})$ scores of the variables that make up the Parkinson's Disease Gravity Index are shown in Table 4.

All the details of the OS results are presented in Table 2. With the OS method, the highest value reached was for the $\mathrm{PsD}$ which is 12.84 , and the lowest ApD is 7.52 (Table 2).

Once the transformation of the scores of the variables studied was carried out using the OS, the PDSI analytical study was carried out. 
TABLE 1: OS model.

\begin{tabular}{lccccc}
\hline MD & $\begin{array}{c}\text { "Corrected value of each } \\
\text { interval" by the OS }\end{array}$ & $\begin{array}{c}\text { Minimum } \\
\text { absolute value }\end{array}$ & $\begin{array}{c}\text { "Corrected value of each } \\
\text { interval" by the OS }\end{array}$ & $\begin{array}{c}\text { Maximum } \\
\text { multiplied by the factor }\end{array}$ & $\begin{array}{c}\text { Maximum } \\
\text { marrected value }\end{array}$ \\
\hline$[3-16]$ & -1.48 & 1.48 & 0 & 0 \\
{$[17-23]$} & -1.01 & & 0.47 & 1.29 \\
{$[24-30]$} & -0.3 & 1.18 & 3.24 & 6.53 \\
{$[31-44]$} & 0.9 & 2.38 & 3.33 & 9.14 \\
{$[45-58]$} & 1.85 & 3.33 & & 9.14 \\
\hline
\end{tabular}

TABle 2: Transformation with the OS.

\begin{tabular}{|c|c|c|c|c|c|c|c|c|}
\hline & & & $\begin{array}{l}\text { "Corrected value of } \\
\text { each interval" by the } \\
\text { OS }\end{array}$ & $\begin{array}{c}\text { Minimum } \\
\text { absolute value }\end{array}$ & $\begin{array}{c}\text { "Corrected value of } \\
\text { each interval" by the } \\
\text { OS }\end{array}$ & Maximum & $\begin{array}{l}\text { Corrected value } \\
\text { multiplied by the } \\
\text { factor }\end{array}$ & Maximum \\
\hline \multirow{6}{*}{$\mathrm{AD}$} & 1 & $36-49$ & -1.85 & \multirow[t]{6}{*}{1.85} & 0 & & 0 & \\
\hline & 2 & $50-63$ & -0.79 & & 1.06 & & 2.91 & \\
\hline & 3 & $64-70$ & -0.6 & & 1.25 & & 3.43 & \\
\hline & 4 & $71-77$ & 0.39 & & 2.24 & & 6.15 & \\
\hline & 5 & $78-84$ & 1.54 & & 3.39 & & 9.3 & \\
\hline & 6 & $85-92$ & 1.68 & & 3.53 & 3.53 & 9.69 & 9.69 \\
\hline \multirow{5}{*}{ MD } & 1 & $3-16$ & -1.48 & \multirow[t]{5}{*}{1.48} & 0 & & 0 & \\
\hline & 2 & $17-23$ & -1.01 & & 0.47 & & 1.29 & \\
\hline & 3 & $24-30$ & -0.3 & & 1.18 & & 3.24 & \\
\hline & 4 & $31-44$ & 0.9 & & 2.38 & & 6.53 & \\
\hline & 5 & $45-58$ & 1.85 & & 3.33 & 3.33 & 9.14 & 9.14 \\
\hline \multirow{6}{*}{$\mathrm{DD}$} & 1 & $0-1.5$ & -1.58 & \multirow[t]{6}{*}{1.58} & 0 & & 0 & \\
\hline & 2 & $1.51-3.01$ & -1.11 & & 0.47 & & 1.29 & \\
\hline & 3 & $3.02-4.52$ & -0.75 & & 0.83 & & 2.28 & \\
\hline & 4 & $4.53-7.54$ & 0.2 & & 1.78 & & 4.88 & \\
\hline & 5 & $7.55-10.56$ & 1.48 & & 3.06 & & 8.4 & \\
\hline & 6 & $10.57-12.07$ & 2.25 & & 3.83 & 3.83 & 10.51 & 10.51 \\
\hline \multirow{7}{*}{$\mathrm{AxD}$} & 1 & $0-1.75$ & -1.23 & \multirow[t]{7}{*}{1.23} & 0 & & 0 & \\
\hline & 2 & $1.76-3.51$ & -0.89 & & 0.34 & & 0.93 & \\
\hline & 3 & $3.52-7.03$ & -0.53 & & 0.7 & & 1.92 & \\
\hline & 4 & $7.04-8.79$ & 0.47 & & 1.7 & & 4.67 & \\
\hline & 5 & $8.80-10.55$ & 0.74 & & 1.97 & & 5.41 & \\
\hline & 6 & $10.56-12.31$ & 1.72 & & 2.95 & & 8.1 & \\
\hline & 7 & $12.32-14.07$ & 2.29 & & 3.52 & 3.52 & 9.66 & 9.66 \\
\hline \multirow{7}{*}{$\mathrm{CD}$} & 1 & $16-27.125$ & -1.71 & \multirow[t]{7}{*}{1.71} & 0 & & 0 & \\
\hline & 2 & $27.126-49.377$ & -1 & & 0.71 & & 1.95 & \\
\hline & 3 & $49.378-60.503$ & -0.12 & & 1.59 & & 4.36 & \\
\hline & 4 & $60.504-71.629$ & 0.66 & & 2.37 & & 6.5 & \\
\hline & 5 & $71.63-82.755$ & 1.25 & & 2.96 & & 8.12 & \\
\hline & 6 & $82.756-93.881$ & 1.39 & & 3.1 & & 8.51 & \\
\hline & 7 & $93.882-105.007$ & 1.65 & & 3.36 & 3.36 & 9.22 & 9.22 \\
\hline \multirow{7}{*}{$\mathrm{ApD}$} & 1 & $0-4.25$ & -1.21 & \multirow[t]{7}{*}{1.21} & 0 & & 0 & \\
\hline & 2 & $4.26-8.51$ & -0.81 & & 0.4 & & 1.1 & \\
\hline & 3 & $8.52-12.77$ & -0.31 & & 0.9 & & 2.47 & \\
\hline & 4 & $12.78-17.03$ & 0.5 & & 1.71 & & 4.69 & \\
\hline & 5 & $17.04-21.29$ & 0.56 & & 1.77 & & 4.86 & \\
\hline & 6 & $21.3-25.55$ & 1.51 & & 2.72 & & 7.46 & \\
\hline & 7 & $25.56-34.07$ & 1.53 & & 2.74 & 2.74 & 7.52 & 7.52 \\
\hline \multirow{8}{*}{ FD } & 1 & $0-3.625$ & -1.33 & \multirow[t]{8}{*}{1.33} & 0 & & 0 & \\
\hline & 2 & $3.626-7.251$ & -0.8 & & 0.53 & & 1.45 & \\
\hline & 3 & $7.252-10.877$ & -0.03 & & 1.3 & & 3.57 & \\
\hline & 4 & $10.878-14.503$ & 0.71 & & 2.04 & & 5.6 & \\
\hline & 5 & $14.504-18.129$ & 0.88 & & 2.21 & & 6.06 & \\
\hline & 6 & $18.13-21.755$ & 1.32 & & 2.65 & & 7.27 & \\
\hline & 7 & $21.756-25.381$ & 1.97 & & 3.3 & & 9.06 & \\
\hline & 8 & $25.382-29.007$ & 2.39 & & 3.72 & 3.72 & 10.21 & 10.21 \\
\hline
\end{tabular}


TABle 2: Continued.

\begin{tabular}{|c|c|c|c|c|c|c|c|c|}
\hline & & & $\begin{array}{c}\text { "Corrected value of } \\
\text { each interval" by the } \\
\text { OS }\end{array}$ & $\begin{array}{c}\text { Minimum } \\
\text { absolute value }\end{array}$ & $\begin{array}{l}\text { "Corrected value of } \\
\text { each interval" by the } \\
\text { OS }\end{array}$ & Maximum & $\begin{array}{l}\text { Corrected value } \\
\text { multiplied by the } \\
\text { factor }\end{array}$ & Maximum \\
\hline \multirow{5}{*}{ NMD } & 1 & $2-16.25$ & -1.29 & 1.29 & 0 & & 0 & \\
\hline & 2 & $16.26-30.51$ & -0.53 & & 0.76 & & 2.09 & \\
\hline & 3 & $30.52-44.77$ & 0.61 & & 1.9 & & 5.21 & \\
\hline & 4 & $44.78-101.81$ & 1.47 & & 2.76 & & 7.57 & \\
\hline & 5 & $101.82-116.07$ & 1.87 & & 3.16 & 3.16 & 8.67 & 8.67 \\
\hline \multirow{5}{*}{ PsD } & 1 & $0-1$ & -0.68 & 0.68 & 0 & & 0 & \\
\hline & 2 & $1.01-2.01$ & 0.39 & & 1.07 & & 2.94 & \\
\hline & 3 & $2.02-4.03$ & 1.1 & & 1.78 & & 4.88 & \\
\hline & 4 & $4.04-5.04$ & 1.84 & & 2.52 & & 6.92 & \\
\hline & 5 & $5.05-8.07$ & 3.89 & & 4.57 & 4.57 & 12.54 & 12.54 \\
\hline \multirow{6}{*}{ SD } & 1 & $0-2.5$ & -1.52 & 1.52 & 0 & & 0 & \\
\hline & 2 & $2.51-5.01$ & -0.81 & & 0.71 & & 1.95 & \\
\hline & 3 & $5.02-7.52$ & 0 & & 1.52 & & 4.17 & \\
\hline & 4 & $7.53-12.54$ & 0.62 & & 2.14 & & 5.87 & \\
\hline & 5 & $12.55-17.56$ & 1.2 & & 2.72 & & 7.46 & \\
\hline & 6 & $17.57-20.07$ & 3.16 & & 4.68 & 4.68 & 12.84 & 12.84 \\
\hline \multicolumn{5}{|c|}{ Index factor creation $(100 / 36.44=2.744)$} & & 36.44 & & 100 \\
\hline
\end{tabular}

AD: age dimension; MD: motor dimension; DD: depression dimension; AxD: anxiety dimension; CD: cognitive dimension; ApD: apathy dimension; FD: fatigue dimension; NMD: nonmotor dimension; PsD: psychosis dimension PsD; SD: sleep dimension.

TABLE 3: Descriptive statistics of the sample $(N=120)$.

\begin{tabular}{|c|c|c|c|c|c|}
\hline Sex & \multicolumn{5}{|c|}{ Male $73(60.8 \%)$} \\
\hline Marital status & \multicolumn{5}{|c|}{$\begin{array}{l}\text { Married } 86(71.7 \%) \\
\text { Single } 10(8.3 \%) \\
\text { Others } 24(20 \%) \\
\end{array}$} \\
\hline \multirow[t]{2}{*}{ Employment status } & \multicolumn{5}{|c|}{$\begin{array}{c}\text { Retired } 73(60.8 \%) \\
\text { Full-time worker } 15(12.5 \%) \\
\text { Housewife or househusband } 12(10 \%) \\
\text { Others } 20(16.7 \%)\end{array}$} \\
\hline & Median & Mean $\pm s d$ & IQR & $S$ & $\bar{K}$ \\
\hline Years of education & 7 & $9.6 \pm 5.2$ & 8 & 0.5 & -0.9 \\
\hline Duration of disease (years) & 8 & $9 \pm 5.6$ & 7 & 1.4 & 3.3 \\
\hline Duration of therapy with L-dopa (years) & 6 & $7.5 \pm 5.3$ & 6.3 & 1.3 & 2.7 \\
\hline Dose of L-dopa (mg/day) & 750 & $683.5 \pm 225.5$ & 250 & -0.1 & 0.5 \\
\hline PIMS & 21 & $19.9 \pm 7$ & 10 & -0.5 & -0.1 \\
\hline CISI total & 10 & $10.1 \pm 4.1$ & 6 & 0.3 & -0.3 \\
\hline
\end{tabular}

$\mathrm{IQR}=$ interquartile range; $S=$ skewness; $C=$ kurtosis; PIMS: Parkinson's Impact Scale; CISI: Clinical Impression of Severity Index for Parkinson's Disease.

TABle 4: Mean \pm sd scores of the variables that make up the Parkinson's Disease Severity Index.

\begin{tabular}{lc}
\hline Age dimension & $68.6 \pm 11.0$ \\
Motor dimension & $28.1 \pm 10.3$ \\
Depression dimension & $5.4 \pm 2.7$ \\
Anxiety dimension & $6.4 \pm 3.6$ \\
Cognitive dimension & $63.3 \pm 18.9$ \\
Apathy dimension & $12.5 \pm 8.9$ \\
Fatigue dimension & $9.6 \pm 6.6$ \\
Nonmotor dimension & $31.6 \pm 18.7$ \\
Psychosis dimension & $1.5 \pm 1.6$ \\
Sleep dimension & $7.6 \pm 4.6$ \\
\hline
\end{tabular}

3.1. Data Quality and Acceptability. There were no missing data; all the information was analyzed. When analysing the items' metric characteristics, we found that PsD has a big floor effect $(61.6 \%)$ and a kurtosis of 3.3 (Table 5).
3.2. Reliability. An appropriate C'a value of 0.71 was gathered, and all items were found to be pertinent to the scale. The item homogeneity index was 0.36 (standard value: $>0.3$ ). Precision evaluated with the SEM $(\mathrm{SEM}=\mathrm{SD} \times \sqrt{ } 1-$ reliability coefficient $=14.5 \times \sqrt{ } 1-0.71=7.8$, a little higher than 7.25 $(14.5 \times 0.5))$ was acceptable.

3.3. Validity. Convergent validity, assessed by Spearman's rho (rhoS) correlation between the PDSteI and the S\&E, was moderately correlated (rhoS: -0.69), with PIMS (rhoS: 0.62) and CISI-PD total (rhoS: 0.58). (Table 6).

The scale's discriminant validity (validity for known groups), assessed among the different stages of the $\mathrm{H} \& \mathrm{Y}$ scale by the Kruskal-Wallis test, showed major significance $\left(X^{2}=32.7, p \leq 0.001\right)$ (Figure 1$)$. 
Table 5: Acceptability data of the variables of the Parkinson's Disease Gravity Index.

\begin{tabular}{lccccccccc}
\hline & Min & Max & Median & Mean & SD & Floor & Ceiling & Skewness & Kurtosis \\
\hline AD & 0 & 9.69 & 3.49 & 5.09 & 2.75 & 6.66 & 4.16 & 0.24 & -0.8 \\
MD & 0 & 9.14 & 3.24 & 3.8 & 2.76 & 10 & 8.33 & 0.42 \\
DD & 0 & 10.51 & 4.88 & 4.24 & 2.85 & 6.66 & 5 & -0.98 \\
AxD & 0 & 9.66 & 1.92 & 3.38 & 2.76 & 9.16 & 4.16 & 0.51 & 0.81 \\
CD & 0 & 9.22 & 6.5 & 5.69 & 2.69 & 2.5 & 3.33 & -0.47 & -0.5 \\
ApD & 0 & 7.52 & 2.47 & 3.32 & 2.76 & 27.5 & 7.5 & -1.22 \\
FD & 0 & 10.21 & 3.57 & 3.65 & 2.75 & 20.83 & 2.5 & 0.18 & 0.22 \\
NMD & 0 & 8.67 & 2.09 & 3.53 & 2.72 & 21.66 & 0.83 & 0.2 & -0.79 \\
PsD & 0 & 12.54 & 0 & 1.87 & 2.78 & 61.66 & 2.5 & -1.3 \\
SD & 0 & 12.84 & 4.17 & 4.17 & 2.76 & 15.83 & 1.66 & 1.69 & 0.16 \\
Total & 12.65 & 77.65 & 36.92 & 38.71 & 14.5 & 0.83 & 1.66 & -0.04 \\
\hline
\end{tabular}

AD: age dimension; MD: motor dimension; DD: depression dimension; AxD: anxiety dimension; CD: cognitive dimension; ApD: apathy dimension; FD: fatigue dimension; NMD: nonmotor dimension; PsD: psychosis dimension; SD: sleep dimension.

Table 6: Correlation between the Parkinson's Disease Gravity Index and other measures.

\begin{tabular}{lcccccccccc}
\hline & Years of disease & Years with L-dopa & L-dopa doses & S\&E & PIMS & CISI1 & CISI2 & CISI3 & CISI4 & CISI total \\
\hline AD & 0.15 & 0.18 & 0.41 & -0.25 & 0.1 & 0.23 & 0.26 & -0.06 & 0.44 & 0.24 \\
MD & 0.5 & 0.55 & 0.52 & -0.76 & 0.56 & 0.76 & 0.72 & 0.67 & 0.52 & 0.82 \\
DD & -0.01 & 0.07 & 0.17 & -0.46 & 0.5 & 0.34 & 0.42 & 0.21 & 0.33 & 0.38 \\
AxD & -0.02 & 0.09 & 0.07 & -0.45 & 0.5 & 0.36 & 0.42 & 0.22 & 0.22 & 0.35 \\
CD & -0.23 & -0.28 & -0.43 & 0.57 & -0.41 & -0.45 & -0.5 & -0.26 & -0.81 & -0.6 \\
ApD & 0.13 & 0.22 & 0.35 & -0.59 & 0.41 & 0.38 & 0.49 & 0.18 & 0.66 & 0.5 \\
FD & 0.1 & 0.2 & 0.27 & -0.58 & 0.46 & 0.43 & 0.48 & 0.19 & 0.59 & 0.5 \\
NMD & 0.17 & 0.26 & 0.34 & -0.42 & 0.46 & 0.3 & 0.36 & 0.19 & 0.26 & 0.32 \\
PsD & -0.07 & 0.03 & 0.12 & -0.31 & 0.2 & 0.2 & 0.27 & 0.08 & 0.22 & 0.2 \\
SD & 0.29 & 0.29 & 0.37 & -0.33 & 0.33 & 0.33 & 0.34 & 0.13 & 0.37 & 0.35 \\
Total & 0.19 & 0.31 & 0.42 & -0.69 & 0.62 & 0.53 & 0.62 & 0.27 & 0.53 & 0.58 \\
\hline
\end{tabular}

$\mathrm{AD}$ : age dimension; MD: motor dimension; DD: depression dimension; AxD: anxiety dimension; CD: cognitive dimension; ApD: apathy dimension; FD: fatigue dimension; NMD: nonmotor dimension; PsD: psychosis dimension; SD: sleep dimension.

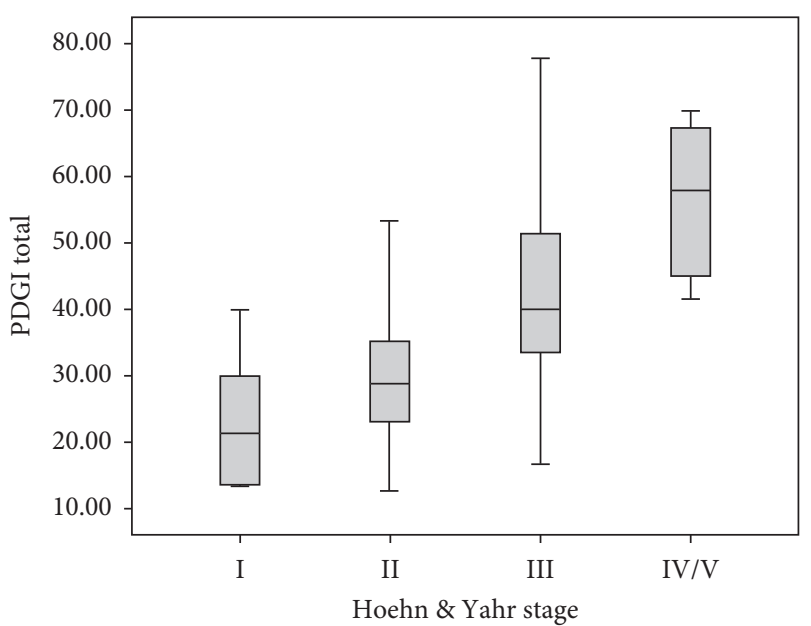

Figure 1: Boxplot of the PDGI total for the H\&Y staging.

\section{Discussion}

In the study, the population was characterized by having a wide representation in stages II and III (34 and 74 patients, respectively) and 9.5 years of disease on average. This is a very common sample in outpatient studies [37].
Regarding the acceptability of the PDGI items, we can point out that, in five items, the floor effect was observed: $\mathrm{ApD}$ (27.5), FD (21.6), $\mathrm{NmD}$ (21.6), PsD (61.6), and SD (15.8). Similar to what was found by Martinez-Martin et al. [37] with their PDCS, the problem of PsD in outpatients is that its prevalence is generally low. For example, Visser et al. [24], using the same evaluation tool, found that only 21 (3\%) of the patients studied had symptoms of this dimension. In the study by Martinez-Martin et al. [37], where the PsD was referred to as hallucinations, they found a floor effect greater than $70 \%$.

None of the patients presented the ceiling effect. As for the asymmetry, only the PsD was outside the norm value (1.6), which evidences its high floor effect/consequent data of its high floor effect. Regarding kurtosis, the CD, AD, and $\mathrm{NmD}$ dimensions had minimum values outside the allowed range $(-1.2,-1.34$, and -1.3 , respectively). The PsD presented a value of 3.3 (Table 5 ).

The corrected item-total correlation ranged from 0.16 for the $\mathrm{AD}$ to 0.72 for the FD. Regarding the C'a, if the item is deleted, then the item that contributes the most to the value of the alpha is the FD; without that item, the alpha fell to 0.61. The item that contributes the least is the CD; when it was removed, the alpha rose to 0.82 . In these circumstances, the range between the alpha values was 0.2 . 
To perform the convergent validity analysis, we found that the AD had generally weak correlations with the variables S\&E, PIMS, and CISI-PD total (values of $-0.25,0.10$, and 0.24 , respectively). The PsD showed similar behaviour, with $d-0.31,0.2$, and 0.2 values, with those same variables (Table 6).

Meanwhile, the $\mathrm{MD}$ reached moderate and strong correlation values: -0.76 against the S\&E, 0.56 with PIMS, and 0.82 with CISI-PD total. The rest of the dimensions had moderate values. The total PDGI was correlated with moderate values: -0.69 with S\&E, 0.62 with PIMS, and 0.58 with CISI-PD total. In the work of Martinez-Martin et al., the PDCS reached values of 0.76 and 0.89 , respectively, compared to PDQ-39, which measures the quality of life and before the CISI-PD total [37].

Compared to other demographic variables such as years of disease and levodopa dose, the PDGI total had moderate correlation values ( 0.37 and 0.46 , respectively). These correlations are like those gained with the PDCS [37] (Table 6).

This PDGI is not considered as a tool for use in daily practice; its function is to be able to carry out a global and therefore extensive evaluation of the situation of a patient.

Overall, our PDGI has adequate metric properties; acceptability, internal consistency, and convergent validity are adequate. This proposal is more in line with a holistic, inclusive evaluation, it does not assume a greater preponderance of motor symptoms, and it includes the different dimensions that affect people with PD. Likewise, it does not allow subjective appreciation to guide giving more or less weight to any of the dimensions, but rather, an objective mathematical algorithm assigns the scores.

\section{Conclusions}

As noted at the beginning, this method allows each dimension to have a weight according to the amount of information it shares with the other variables and dimensions. This is the method's advantage over existing scales, which do not give values of different scales gathered under a certain category such as mild or severe. The PDGI groups the scores that share information about the subject. Therefore, each score offers part of the clinical situation of a patient, and this allows for a detailed summation of the patient's state.

\section{Data Availability}

The data used to support the findings of this study are available from the corresponding author upon request.

\section{Ethical Approval}

This study was approved by the Teaching and Research Department of the Carlos Andrade Marín Hospital and by the Bioethics Committee of the University of Navarra (Spain).

\section{Consent}

All the patients gave their informed consent to participate in the study.

\section{Disclosure}

This research had no kind of funding, nor was it carried out as part of the work in the institutions in which the authors work. It is part of doctoral thesis research. All the inherent costs of it have been covered by the principal investigator (MS-D).

\section{Conflicts of Interest}

The authors declare that they have no conflicts of interest.

\section{References}

[1] M. J. Armstrong and M. S. Okun, "Diagnosis and treatment of Parkinson disease: a review," JAMA, vol. 323, no. 6, pp. 548-560, 2020.

[2] R. Cacabelos, "Parkinson's disease: from pathogenesis to pharmacogenomics," International Journal of Molecular Sciences, vol. 18, no. 3, p. 551, 2017.

[3] E. De Pablo-Fernández, A. J. Lees, J. L. Holton, and T. T. Warner, "Prognosis and neuropathologic correlation of clinical subtypes of Parkinson disease," JAMA Neurology, vol. 76, no. 4, pp. 470-479, 2019.

[4] J. M. Beitz, "Parkinson's disease: a review," Frontiers in Bioscience, vol. 6, no. 1, pp. 65-74, 2014.

[5] K. Ray Chaudhuri, J. M. Rojo, A. H. V. Schapira et al., "A proposal for a comprehensive grading of Parkinson's disease severity combining motor and non-motor assessments: meeting an unmet need," PLoS One, vol. 8, no. 2, pp. e57221-e57227, 2013.

[6] P. Martinez-Martin, C. Rodriguez-Blazquez, K. Abe et al., "International study on the psychometric attributes of the non-motor symptoms scale in Parkinson disease," Neurology, vol. 73, no. 19, pp. 1584-1591, 2009.

[7] M. Hoehn and M. Yahr, "Parkinsonism: onset, progression, and mortality," Neurology, vol. 57, no. 10, pp. S11-S26, 1967.

[8] F. Stocchi, F. G. Radicati, K. R. Chaudhuri et al., "The Parkinson's disease composite scale: results of the first validation study," European Journal of Neurology, vol. 25, no. 3, pp. 503-511, 2018.

[9] S. Stevens, "On the theory of scales of measurement," Science, vol. 103, no. 2684, pp. 677-680, 1946.

[10] R. A. Fisher, Statistical Methods for Research Workers, Oliver and Boyd Ltd, Edinburgh, Scotland, 5th edition, 1934.

[11] F. W. Young, "Quantitative analysis of qualitative data," Psychometrika, vol. 46, no. 4, pp. 357-388, 1981.

[12] F. W. Young, "Scaling," Annual Review of Psychology, vol. 35, no. 1, pp. 55-81, 1984.

[13] C. Eckart and G. Young, "The approximation of one matrix by another of lower rank," Psychometrika, vol. 1, no. 3, pp. 211-218, 1936.

[14] F. W. Young, J. de Leeuw, and Y. Takane, "Regression with qualitative and quantitative variables: an alternating least squares method with optimal scaling features," Psychometrika, vol. 41, no. 4, pp. 505-529, 1976.

[15] F. W. Young, Y. Takane, and J. de Leeuw, "The principal components of mixed measurement level multivariate data: an alternating least squares method with optimal scaling features," Psychometrika, vol. 43, no. 2, pp. 279-281, 1978.

[16] A. S. Beavers, J. W. Lounsbury, J. K. Richards, S. W. Huck, G. J. Skolits, and S. L. Esquivel, "Practical considerations for using exploratory factor analysis in educational research," 
Practical Assessment, Research \& Evaluation, vol. 18, no. 6, pp. 1-13, 2013.

[17] W. R. Gibb and A. J. Lees, "The relevance of the Lewy body to the pathogenesis of idiopathic Parkinson's disease," Journal of Neurology, Neurosurgery \& Psychiatry, vol. 51, no. 6, pp. 745-752, 1988.

[18] J. Marinus, M. Visser, A. M. Stiggelbout et al., "A short scale for the assessment of motor impairments and disabilities in Parkinson's disease: the SPES/SCOPA," Journal of Neurology, Neurosurgery \& Psychiatry, vol. 75, no. 3, pp. 388-395, 2004.

[19] A. S. Zigmond and R. P. Snaith, "The hospital anxiety and depression scale," Acta Psychiatrica Scandinavica, vol. 67, no. 6, pp. 361-370, 1983.

[20] M. Serrano-Dueñas, M. Serrano, D. Villena, and D. Granda, "Validation of the Parkinson's disease-cognitive rating scale applying the movement disorder society task force criteria for dementia associated with Parkinson's disease," Movement Disorders Clinical Practice, vol. 4, no. 1, pp. 51-57, 2016.

[21] M. Serrano-Dueñas, P. Martínez-Martín, T. Merchán, R. Bravo, and M. Serrano, "Properties of the Apathy scale (AS) for use on Parkinson's patients," Advances in Parkinson's Disease, vol. 2, no. 2, pp. 53-57, 2013.

[22] J. D. Fisk and S. E. Doble, "Construction and validation of a fatigue impact scale for daily administration (D-FIS)," Quality of Life Research, vol. 11, no. 3, pp. 263-272, 2002.

[23] K. R. Chaudhuri, P. Martinez-Martin, R. G. Brown et al., "The metric properties of a novel non-motor symptoms scale for Parkinson's disease: results from an international pilot study," Movement Disorders, vol. 22, no. 13, pp. 1901-1911, 2007.

[24] M. Visser, D. Verbaan, S. M. Van Rooden, A. M. Stiggelbout, J. Marinus, and J. J. Van Hilten, "Assessment of psychiatric complications in Parkinson's disease: the SCOPA-PC," Movement Disorders, vol. 22, no. 15, pp. 2221-2228, 2007.

[25] J. Marinus, M. Visser, J. J. van Hilten, G. J. Lammers, and A. M. Stiggelbout, "Assessment of sleep and sleepiness in Parkinson disease," Sleep, vol. 26, no. 8, pp. 1049-1054, 2003.

[26] M. M. Hoehn and M. D. Yahr, "Parkinsonism: onset, progression, and mortality," Neurology, vol. 17, no. 5, pp. 427-442, 1967.

[27] R. S. E. A. Schwab, "Projection techniques for evaluating surgery in Parkinson's disease," in Proceedings of the Third Symposium on Parkinson's Disease, pp. 152-157, Royal College of Surgeons in Edinburgh, Edinburgh, UK, May 1968.

[28] S. Calne, M. Schulzer, E. Mak et al., "Validating a quality of life rating scale for idiopathic Parkinsonism: Parkinson's impact scale (PIMS)," Parkinsonism \& Related Disorders, vol. 2, no. 2, pp. 55-61, 1996.

[29] P. Martínez-Martín, C. Rodríguez-Blázquez, M. J. Forjaz, and J. de Pedro, "The clinical impression of severity index for Parkinson's disease: international validation study," Movement Disorders, vol. 24, no. 2, pp. 211-217, 2009.

[30] H. A. Sturges, "The choice of a class interval," Journal of the American Statistical Association, vol. 21, no. 153, pp. 65-66, 1926.

[31] C. A. McHorney and A. R. Tarlov, "Individual-patient monitoring in clinical practice: are available health status surveys adequate?" Quality of Life Research, vol. 4, no. 4, pp. 293-307, 1995.

[32] J. C. Hobart, A. Riazi, D. L. Lamping, R. Fitzpatrick, and A. J. Thompson, "Improving the evaluation of therapeutic interventions in multiple sclerosis: development of a patientbased measure of outcome," Health Technology Assessment, vol. 8, no. 9, pp. 1-48, 2004.
[33] N. Aaronson, J. Alonso, A. Burnam et al., "Assessing health status and quality-of-life instruments: attributes and review criteria," Quality of Life Research, vol. 11, no. 3, pp. 193-205, 2002.

[34] R. Fitzpatrick, J. M. Norquist, and C. Jenkinson, "Distribution-based criteria for change in health-related quality of life in Parkinson's disease," Journal of Clinical Epidemiology, vol. 57, no. 1, pp. 40-44, 2004.

[35] G. R. Norman, J. A. Sloan, and K. W. Wyrwich, "Interpretation of changes in health-related quality of life the remarkable universality of half a standard deviation," Medical Care, vol. 41, no. 5, pp. 582-592, 2003.

[36] H. Akoglu, "User's guide to correlation coefficients," Turkish Journal of Emergency Medicine, vol. 18, no. 3, pp. 91-93, 2018.

[37] P. Martinez-Martin, F. G. Radicati, C. Rodriguez Blazquez et al., "Extensive validation study of the Parkinson's disease composite scale," European Journal of Neurology, vol. 26, no. 10, pp. 1281-1288, 2019. 